\title{
Organizational Model of Big Data Innovation Alliance: Case Analysis of Virtual Cluster Perspective
}

\author{
Xiaomei Wang and Tianzhu $\mathrm{Li}^{*}$ \\ School of Business Administration, Liaoning University of Science and Technology, Anshan City, Liaoning Province, \\ China
}

\begin{abstract}
In recent years, the Big Data Innovation Alliance has received widespread attention and rapid development, but its effective organizational model remains to be further revealed. From the perspective of industrial clusters, the Big Data Innovation Alliance can be seen as an industrial agglomeration phenomenon. Virtual clusters and big data industry innovation alliances have similarities in cross-industry, cross-regional, and diversified features. Therefore, studying the organizational model of the Big Data Innovation Alliance based on the perspective of virtual clusters is a new exploration direction. Based on this, the Zhongguancun Big Data Industry Alliance is taken as an example to select the two research variables of the constituent elements and organizational characteristics for a single case study, in an attempt to draw theoretical implications for the organizational model of the Big Data Innovation Alliance.
\end{abstract}

Keywords - innovation alliance; virtual cluster; constituent elements; organizational characteristics; policy recommendations

\section{INTRODUCTION}

Judging from the current development status of the big data industry, the industry is growing fast and the scale is expanding. However, it is rarely reported that the big data can be effectively used for technological innovation. The bottleneck is that it is difficult to effectively connect, mine and utilize data of different dimensions. In this context, innovative alliances with multiple characteristics such as resource integration and complementary advantages have become important forms of big data innovation. The technological innovation activities of the big data industry not only need to be able to realize the internal driving force and operation mechanism of technological innovation, but also require the policies and financial support of auxiliary institutions such as the government and financial institutions to realize the synergy development and innovation in the true sense. Therefore, exploring the organizational model of the Big Data Innovation Alliance, including the main structure of the innovation alliance, the allocation of powers and the integration of various resources, has strong practical significance. By collating the literature, the author finds that the domestic research on the organizational model of innovation alliance mainly focuses on the classification of organizational models, the influencing factors of organizational models, and the theoretical construction of organizational models ${ }^{[1][2][3]}$. The rare literature studies the organizational model of the Big Data Innovation Alliance.

From the perspective of industrial clusters, the Big Data Innovation Alliance can be seen as a phenomenon of industrial agglomeration based on big data. The cross-industry and cross-regional characteristics of the big data industry make the traditional industrial cluster model unable to meet the specific requirements of the big data innovation alliance organization model. Virtual clusters break the spatial and geographical constraints of traditional industrial clusters, and better promotes the integration of resources in enterprises, in terms of technology, resources, and knowledge learning, which provides a new perspective for the organization model of big data innovation alliance. Based on this perspective, this paper selects two research variables, the constituent elements and organizational characteristics, to conduct a single case study on the Zhongguancun Big Data Industry Alliance, and attempts to obtain theoretical inspiration for the organizational model of the Big Data Innovation Alliance.

\section{Virtual Cluster Features Of Big Data INNOVATION ALLIANCE}

The Big Data Innovation Alliance is an organizational form in which the relevant companies and institutions in the big data industry are guided by government policies and are guaranteed by certain regulations or contracts. Based on the foregoing, the relevant innovation entities of the Big Data Innovation Alliance are scattered and concentrated in the geographical distribution. In essence, each innovation entity forms a virtual big data industry cluster within the innovation alliance. Based on this, the author believes that the Big Data Innovation Alliance has the following four virtual cluster features ${ }^{[4][5]}$.

(1) $1+1>2$.The member unit of a virtual cluster is an independent enterprise. There is no affiliation, and the coupling of virtual clusters enables the member units in the industrial cluster to cooperate and produce economic benefits that individuals cannot bring alone. For the Big Data Innovation Alliance, which is composed of a number of complementary enterprises with the same objectives and the same interests, the property rights of each member unit 
are also relatively independent. The virtualization of the Big Data Innovation Alliance maximizes the integration of resources across all parties. Based on this, we can think that the integration effect of virtual clusters is consistent with the synergistic innovation effect of $1+1>2$ generated by the multi-agent of the Big Data Innovation Alliance.

(2) Boundary ambiguity. The cross-regional agglomeration of virtual clusters breaks the boundary constraints of traditional industrial clusters, blurs and dynamics the boundaries of industrial clusters, and circumvents the external uneconomic phenomena that traditional industrial clusters may generate in the same region. The industry characteristics of big data determine that the members of the Big Data Innovation Alliance cover a wide range of industries and geographical locations. It can be seen from this that the Big Data Innovation Alliance is a virtual clustered technology innovation alliance. The extensive types and distribution of the members of the Big Data Innovation Alliance can expand the scale of the industry, and form a big data industry cluster with a complete industrial chain to better realize the technological innovation activities of the big data industry.

(3) Information network. Like the virtual cluster, the Big Data Innovation Alliance can effectively network similar industries and related enterprises and institutions in one organization. The information sharing among enterprise units effectively enhances the development of economies of scale and scope. On the one hand, the operating model established by the members of the Big Data Innovation Alliance based on network communication technology can save the transaction cost of the entity and increase the flexibility of the big data innovation alliance to respond to the market; on the other hand, the member units of the Big Data Innovation Alliance can also make full use of the advantages of network to carry out brand promotion, forming a broader and lasting brand effect.

(4) Knowledge spillover. Knowledge spillover refers to the ability of other member units of a virtual cluster to quickly learn innovative knowledge of successful enterprises through imitative and innovative means when the technological innovation activities of a member of the cluster are successful, and form a knowledge spillover effect within the cluster. The Big Data Innovation Alliance has formed an industrial scope phenomenon in which a certain leading enterprise is the core and a large number of related enterprises gather together. This technological innovation model not only increases the convenience of mutual learning among member units, but also enhances the ability and efficiency of member units' independent innovation and market intelligence and market information. Ability and efficiency in obtaining market intelligence and market information. Therefore, we can think that the Big Data Innovation Alliance also has the knowledge spillover principle of virtual clusters.

\section{RESEARCH DESIGN}

\section{A. Research Variable}

Based on the organizational model of industrial clusters, this paper defines the organizational model of big data innovation alliance as: It will gather a large number of cross-domain and industry-restricted and highly concentrated related enterprises and institutions, and use scientific and rational organization of division of labor and market distribution to achieve an industrial organization form that saves production costs. The research variables of organizational patterns vary from different research perspectives. Based on the perspective of virtual clusters, this paper argues that the organizational model of the Big Data Innovation Alliance can be analyzed based on the following research variables.

(1) Component. The element refers to the smallest unit that constitutes the existence of an objective thing and maintains its movement, and is an indispensable phenomenon that constitutes a thing ${ }^{[6]}$.According to the interpretation of modern Chinese, the constituent elements refer to the indispensable factors that constitute the matter and the basic unit of the system. From the perspective of virtual clusters, the components of the Big Data Innovation Alliance mainly include common goals, membership, operational mechanisms, economic links and network protocols.

(2) Organizational characteristics. According to the understanding of modern Chinese, effective organization of characteristics includes clear goals, related skills, and good communication. Starting from the virtual cluster characteristics of Big Data Innovation Alliance, this paper analyzes the organizational characteristics of Big Data Innovation Alliance from two aspects: organizational structure, function and organizational form.

\section{B. Research Methods and Data Sources}

The purpose of the case study is to generate theory based on case-based induction, so it is a powerful tool for constructing theory ${ }^{[7]}$. As a commonly used qualitative research method, case studies are suitable for studying the "how" and "why" problems ${ }^{[8]}$. This paper is a preliminary exploration of the organizational model of big data innovation alliance based on the perspective of virtual industry clusters. It just happens to be a question of "how" and "why", so this paper uses a single case study.

The paper selects the Zhongguancun Big Data Industry Alliance (hereinafter referred to as "Zhongguancun Alliance") as a typical case mainly based on two considerations. On the one hand, more than $60 \%$ of the 500 member companies of the Zhongguancun Alliance are well-known big data companies in China. In China's top ten most influential big data social think tanks, the Zhongguancun Alliance became the first alliance organization to enter the list with a news media influence of 8.17, media influence of 5.88, and a comprehensive score of 7.02. On the other hand, the cooperation units of the 
Zhongguancun Alliance are spread all over the country. For example, the Zhongguancun Alliance has reached a cross-domain strategic cooperation intention with relevant organizations and enterprises such as Silicon Valley, the United Kingdom, South Korea, and France, and has formed a spatial agglomeration form that spans geographical restrictions and industry restrictions. This phenomenon is consistent with the clustering characteristics of virtual clusters.

Sources of data commonly used in case studies include literature, archival records, interviews, direct observations, participatory observations, and physical evidence ${ }^{[9]}$.The extensiveness and particularity of the distribution of Zhongguancun alliance enterprises have made it impossible to conduct interviews and field visits in specific studies. Therefore, the access to the case data in this article mainly includes the official website, news media reports and official public number push. At the same time, the author continuously expands the scope of data collection according to the actual situation in the research process, and obtains relevant evidence support as comprehensively as possible.

\section{Case Introduction}

The Zhongguancun Alliance is an international, industry-based, non-profit industry organization that is voluntarily composed of big data-related enterprises, institutions, and social organizations in China. Its founding purpose is to improve the external environment of the enterprise and guide the strategic decision of the enterprise by pooling multiple resources, and finally promote the transformation and upgrading of the real economy by constructing a service platform that helps the enterprise grow.Based on the principle of "openness, sharing, cooperation and win-win”, Zhongguancun Alliance gathers resources from politics, production, learning and research, and has won the recognition and trust of many big data leaders and experts. The reason why the Zhongguancun Alliance can operate successfully is the three concepts of the Zhongguancun Alliance. The first concept is the "think tank". The Zhongguancun Alliance has established the operation mode of "system production machine + theoretical porter + talent cultivation place", and it is the duty to make suggestions and suggestions by gathering many industry leaders; the second concept is "media "The use of third-party media and the alliance's own public number to integrate the advanced thinking of big data into all aspects of the operation of the Zhongguancun Alliance; The third concept is "capital". The most prominent of the Zhongguancun Alliance in the financing process is the adherence to the aircraft carrier investment theory. That is to say, the company that first invests in the aircraft carrier class, and then invests in the battleship-type and cruiser-type enterprises together with the aircraft carrier, and finally forms a fleet to develop. The Zhongguancun Alliance fully utilizes the work platform of the government science and technology association service innovation development, and strengthens the close cooperation between the alliance itself and the institutes, associations, universities and scientific research institutions. At the same time, it links the upstream and downstream enterprises of the big data industry and opens up the communication barriers of the big data industry. It brings together think tanks from various industries and fields to realize mutual benefit and win-win between industrial resources and intellectual resources.

\section{CASE STUdY}

\section{A. The Components of the Big Data Innovation Alliance}

From the perspective of virtual clusters, the components of the Big Data Innovation Alliance mainly include common goals, membership, operational mechanisms, economic links and network protocols.

(1) Common goals. A goal in a virtual cluster is a guide to the operation of a business. Guided by common collective goals, arrange the resources necessary to achieve the strategy and coordinate the economic behavior of all parties involved ${ }^{[10]}$.According to the case study, the fundamental focus of the Zhongguancun Alliance's goal is to bring together the leading resources of each segment of the industry, to improve the external environment of the enterprise to help the growth of the enterprise, and ultimately to achieve industrial upgrading throughout the country. For example, the Zhongguancun Alliance systematizes and structures the country's big data strategy, and builds a unique "ecological management theory", which aggregates industries into different industrial ecosystems according to different categories, and helps various industries to create eco-enhanced core enterprises. In another example, the Zhongguancun Alliance participates in the local government's big data industry planning, and applies big data thinking to urban operations, public safety, culture, and consumption through activities such as the Guiyang Digital Expo and the Big Data 100-themed salon. It can be seen from this that the goal of the Big Data Innovation Alliance is to coordinate and integrate all resources to achieve the basis of big data technology innovation activities, which is consistent with the role of the common goal of the virtual cluster.

(2) Member composition. Virtual clusters are aggregates of cross-regional and cross-industry formed by relevant enterprises and institutions using information and network technologies. They break through the geographical limitations of traditional industrial clusters and make their membership more diversified and complicated. According to the case study of the Zhongguancun Alliance, the Zhongguancun Alliance has nearly 500 member companies, and has established 15 industry special committees, 5 industry research institutes, and 7 local alliances nationwide. Second, the Zhongguancun Alliance In terms of international development has reached strategic cooperation intentions with relevant institutions and enterprises such as the United Kingdom, the United States, and South Korea, and has made positive contributions to promoting cross-industry and cross-regional development of the big data industry. Therefore, from the perspective of membership, the Big Data Innovation Alliance is similar to 
the members of the virtual cluster, and member units can achieve technological innovation activities across geographical restrictions.

(3) Operating mechanism. In the partner selection mechanism, all enterprises, institutions, research institutes, and organizations that are engaged in the field of big data voluntarily submit applications and actively abide by the articles of association. After paying membership fees, they can become members of the Zhongguancun Alliance. The member units of the level enjoy the corresponding membership rights and bear the corresponding membership obligations. In terms of organizing the division of labor mechanism, the Zhongguancun Alliance divides the member units into five functional departments, and each department has clear division of labor and mutual supervision, which ensures the efficient operation of the Zhongguancun alliance. Therefore, from the perspective of virtual cluster analysis, it is known that the continuous operation of Big Data Innovation Alliance needs not only the power of technological innovation, but also the partner selection mechanism, organizational division mechanism, risk sharing mechanism, supervision and management mechanism, etc. The operational mechanism in place will effectively restrain, coordinate and motivate the behavior of the members of the Big Data Innovation Alliance, which is more conducive to the realization of the common goal of the Big Data Innovation Alliance.

(4) Economic connection. The Zhongguancun Alliance is committed to promoting the integration of industrial ecology and big data, the integration of traditional industries and emerging industries, and the integration of academic institutions and industry. Through the convergence of four types of enterprises, governments, universities and research institutions, production and financial services institutions, the final construction A collaborative development of the big data industry ecosystem. It can be seen from this that the Big Data Innovation Alliance is not a simple superposition of similar enterprises, but a series of leading enterprises in the field of big data and other specialized enterprises and institutions through a certain information technology to form an organic connected virtual enterprise agglomeration. In this paper, the author believes that the Big Data Innovation Alliance is a loose network organization, and contractual connections dominate.

(5) Network protocol. The network protocol of a virtual cluster refers to the conventions and guidelines that must be observed by all parties in the cluster to achieve resource sharing and complementary advantages. According to the case study, the "Zhongguancun Big Data Industry Alliance Charter" promulgated by the Zhongguancun Alliance clearly stipulates the membership joining and exit conditions, the principle of interest distribution, and the organizational division of members. The member units of the Zhongguancun Alliance should jointly build the overall market brand image of China's big data industry and enhance the global competitiveness and national discourse power of China's big data industry on the basis of adhering to the charter system. According to this, the network agreement of the Big Data Innovation Alliance refers to the binding documents such as the charter that the members of the Big Data Innovation Alliance must abide by. It clearly defines the rights and obligations of member units and is the binding force of the members of the Big Data Innovation Alliance.

\section{B. Organizational Characteristics of the Big Data Innovation Alliance}

This paper analyzes the organizational characteristics of the Big Data Innovation Alliance from the aspects of organizational structure, function and organizational form.

(1) Organizational structure and function. Organizational structure generally refers to the dynamic structure system formed by all members of the organization to achieve professional division of work in terms of the organization's objectives, duties, and rights. The organizational structure and functions of the Big Data Innovation Alliance should be analyzed from the following three aspects.

1) Sponsor. The initiator of a virtual cluster can be undertaken by a company, an organization, or an individual. From the perspective of the virtual cluster analysis, the founder of the Zhongguancun Alliance is an enterprise, and its organization is mainly enterprise-oriented. For the enterprise-led Big Data Innovation Alliance, the various rules and regulations of the Big Data Innovation Alliance are formulated by leading companies to conduct technological innovation activities by leading the company's technical standards.

2) Virtual cluster. Through case studies, it is known that the Zhongguancun Alliance is a coalition organization, which established by leading enterprises in the field of big data, universities and research institutions, production, financial service institutions and other relevant subjects, supported by government policies, with legally binding contracts. organization. The regulations of the Zhongguancun Alliance stipulate that a big data sharing patent pool between member units should be established, and a big data transaction and information resource sharing exchange platform should be established. It can be seen from this that the Big Data Innovation Alliance is a related enterprise and institution with independent and geographically dispersed property rights. Based on the "organizational proximity", the "psychological distance" and "space distance" between member units are reduced, and the member units are regulated. The core competency to participate in the virtual cluster of the big data industry for virtual enterprise operations.

3) Virtual enterprise. In order to achieve a certain goal, a virtual enterprise is a relatively close temporary network alliance that is conditionally selected by some enterprises in the best dynamic combination ${ }^{[11]}$.According to the case study, the Zhongguancun Alliance not only contains domestic well-known big data enterprises, but also related enterprises and institutions distributed along the industrial chain. It is the most advantageous innovation subject 
selected by the Zhongguancun Alliance to achieve common goals. Based on this, it can be seen that the virtual enterprise of the Big Data Innovation Alliance can effectively connect with each member unit. When the Big Data Innovation Alliance needs to realize technological innovation activities, it will quickly integrate the original dispersed enterprise resources according to market demand to form an efficient collaborative innovation system. The virtual enterprise of the Big Data Innovation Alliance includes both virtual enterprises in the big data industry and virtual enterprises in the general sense.

(2) Organizational form. Organizational form generally reflects the division of labor and cooperation between members of the organization. According to the case study, the Zhongguancun Alliance uses the alliance charter as a binding document, and organizes affiliate members to declare government research projects in the field of big data, and conduct joint research and research and development on common technologies and standards in the field of big data.The Zhongguancun Alliance further promotes the synergy between industry, academia and research by promoting cooperation between upstream and downstream of the internal industry chain, thereby building a flexible and efficient big data industry ecosystem.The Zhongguancun Alliance builds a big data transaction and resource sharing platform by establishing a data sharing patent pool among member units and setting up a big data incubation fund for start-up projects in the big data field. And cooperate with universities and scientific research institutions to carry out talent training and joint recruitment to form China's intellectual property in the field of big data.

1) The members of the Big Data Innovation Alliance have a shared vision, which aims to foster a learning environment for the entire organization and give full play to the creative thinking of member companies to create a continuous, flat management structure. By reducing the management level, increasing the management scope, and compressing functional departments and organizations, the Big Data Innovation Alliance can reduce the intermediate management level between the decision-making layer and the execution layer as much as possible, so that the decision of the Big Data Innovation Alliance can be as fast as possible. Delivered to each node enterprise to achieve a flexible new management model.

2) The collaborative innovation development of the members of the Big Data Innovation Alliance includes both competition and cooperation. In order to compete for better resources among homogeneous enterprises, member units need to continuously learn to improve their independent innovation capabilities and gain opportunities to share with other high-quality enterprise resources. Based on this, it is seen that the members of the Big Data Innovation Alliance need to continuously strengthen their learning ability, and the essential feature of the learning organization is to be good at continuous learning.

3) From the case study, the leader of the Big Data Innovation Alliance has transformed from a single power source role to a diversified leading role. Just like the EMU theory of management, the locomotive of the Big Data Innovation Alliance is no longer the driving force of the whole car, and more is responsible for leading the direction of the whole car. Leaders of the Big Data Innovation Alliance need to design a basic concept for the development of big data innovation alliances based on common goals, and strengthen the understanding of the Big Data Innovation Alliance's member units on the way the Big Data Innovation Alliance operates, This will better promote the integration of various resources within the Big Data Innovation Alliance.

\section{TheOretical Findings And Policy RECOMMENDATIONS}

\section{A. Theoretical Discovery}

Based on the aforementioned case studies, the following theoretical findings are further obtained.

(1) From the perspective of constituent elements, modern information technology, common goals and good operational mechanisms are necessary conditions for the Big Data Innovation Alliance to obtain maximum economic benefits. Members of the Big Data Innovation Alliance can ensure the smooth flow of information within the Big Data Innovation Alliance by strengthening website construction, proxy communication technology, and internal coordination technologies. On the other hand, the Big Data Innovation Alliance pays attention to the formulation of rules and regulations, and establishes the trust mechanism and benefit distribution mechanism of member companies to coordinate the relationship between various innovation entities, thus ensuring the successful operation of the Big Data Innovation Alliance.

(2) From the perspective of organizational characteristics, the Big Data Innovation Alliance adopts a flat management structure. The post of the internal functional departments of the Big Data Innovation Alliance is lean and efficient, and the responsibility is matched. The management level of each innovation subject of the Big Data Innovation Alliance is reduced, and the degree of specialization and work efficiency of the internal management of the Big Data Innovation Alliance is improved. For the learning organization of the Big Data Innovation Alliance, a clear division of labor can make each member unit more focused on their own work, and improve the efficiency of work and innovation while also contributing to the accumulation and knowledge of individual experience of member units. The perfection enables the Big Data Innovation Alliance to form a comprehensive performance effect that is higher than the sum of individual performance.

\section{B. Policy Suggestion}

Judging from the virtual cluster characteristics of the Big Data Innovation Alliance, the future development of the Big Data Innovation Alliance organization model should be based on the principle of promoting economic development and industrial transformation and upgrading, and helping the 
big data industry of the whole society to land. It is necessary to take into account both the external environment and the internal environment of the Big Data Innovation Alliance, and focus on the following points.

(1) Organizational pattern design based on external environment

1) Improve the data management mechanism and improve the top-level design of the government.

The difference between the Big Data Innovation Alliance and the traditional industrial technology innovation alliance lies in the particularity of the big data industry itself. The huge amount of information and fast running speeds lead to data fragmentation, data silos and security privacy problems in the application process of big data. Therefore, for the technological innovation activities of big data. On the one hand, government departments should strengthen the top-level design of the government system, improve the efficiency of the Big Data Innovation Alliance and other relevant institutions in deploying resources and solving problems, and attach importance to the establishment of a collaborative innovation platform for the big data industry. On the other hand, government departments should support the members of the Big Data Innovation Alliance to establish a privacy protection system for the big data industry, encourage the whole society to participate in big data collection and big data governance, and strengthen the whole society in big data professionals and big Cultivation of data thinking.

2) Accelerate the integration of innovative resources and promote industrial upgrading.

At present, the overall structure of China's industrial development is not balanced. Many small and medium-sized enterprises, even large enterprises in certain industries, cannot establish their own information collection mechanisms or information collection platforms. They are not sensitive enough to the country's policies and regulations and market environment changes, and cannot cope with the market. Risks and losses from environmental changes. The core of big data is "forecasting." Therefore, under the support of government policies, the Big Data Innovation Alliance should accelerate the integration of information resources of the government and related institutions, and unite government departments to accelerate the infrastructure construction of the big data industry. At the same time, strengthen cooperation with universities and research institutions to predict changes in the future market through big data technology innovation activities, help small and weak enterprises to break a single business model and achieve structural upgrading of the entire industry.

(2) Organizational model design based on internal environment

1) Strengthen virtual cluster collaboration and build a clustered innovation network.
From the case of this article, the technology and cooperation standards of enterprise-led innovation alliances are dominated by leading enterprises. Once the status of leading enterprises declines, the original technical standards may be changed, which may increase the cost input of member companies. Therefore, the organizational model of the Big Data Innovation Alliance will adopt virtual cluster-based technology innovation in the future development, and consider adding third-party virtual enterprise evaluators. The virtual enterprise evaluator can make judgments on the independent innovation ability and competitiveness of the member units entering the Big Data Innovation Alliance, and track and evaluate the performance and competitiveness of the alliance.

2) Reform existing management systems and build learning organizations.

A sound management system is a prerequisite for the Big Data Innovation Alliance to continue to operate. Therefore, the future development of the big data innovation alliance organization model needs to adopt a flat management structure, reduce the management level within the alliance as much as possible, and increase the management scope; On the other hand, a learning mechanism is established within the Big Data Innovation Alliance. Strengthen the knowledge transfer effect among the members of the Innovation Alliance, and make full use of the government's macro guidance, market guidance of leading enterprises, resource sharing of universities and scientific research institutions, production of service organizations, investment guarantees, etc. to promote cross-industry and cross-regional innovation of the Big Data Innovation Alliance. Cooperation to achieve multi-agent collaborative innovation activities in the big data industry.

\section{RESEARCH OUTLOOK}

This paper is based on the virtual cluster perspective on the organizational model of big data innovation alliance. There are still some shortcomings: First, this paper belongs to single case study, which helps to analyze complex phenomena in depth, and the author also in the research process. A variety of data sources have been used for data collection and verification, but the promotion and universality of the research conclusions still have insufficient persuasiveness. Therefore, the author will further use multi-case analysis to verify the validity of the research conclusions in the next study. Second, based on the author's current theoretical grasp, this paper may not be comprehensive and rigorous in the selection of research variables. Subsequent research needs to enrich the selection and elaboration of research variables as much as possible, and further analyze the generality and particularity of the Big Data Innovation Alliance in terms of research variables according to the characteristics of big data innovation, and provide the future development of the big data innovation alliance organization model. More general theoretical implications. 


\section{ACKNOWLEDGMENT}

This work was financially supported by the National Natural Science Foundation of China "Research on the Rescue Mechanism of Technological Innovation Failure: Taking the Biopharmaceutical Industry as an Example” (No. 71772082), "Research on the Evaluation and Distribution of Big Data Value in Relay Innovation” (No. 71721080) and "Model and Empirical Application Research of Relay Innovation: Taking Biopharmaceuticals as an Example” (No. 71372121).

\section{REFERENCES}

[1] Sun Liang, Li Jianling, Li Daisong. Organizational Model and Government Role of Industrial Technology Innovation Alliance [J]. China Science and Technology Forum, 2015,(3): 12-17.

[2] Wang Huizhen, Sun Minggui. Research on Influencing Factors of Technology Alliance[J]. Science \& Technology Progress and Policy, 2010, 27(12): 6-9.

[3] Li Yongkui, Le Yun, Zhang Yan, Hu Yi. China's major engineering organization model under the dual role of "government-market": theory-based theory construction[J]. Journal of Systems Management, 2018, 27(1): 147-156.

[4] Xiao Jianhua, Zhang Liufang, Sun Ling. Research on the Governance Model and Synergistic Effect of Virtual Industrial Clusters in China: Taking the Service Industry as an Example [J]. Science and Technology Progress and Policy, 2016, 33(15): 44-49.

[5] Zhou Jinbo, Cai Chengfeng. Research on High-tech Industry Cluster Model__ Taking Guilin High-tech Industrial Development Zone as an Example[J]. Journal of Liuzhou Vocational and Technical College, 2012, 12(5): 10-14.

[6] Liu Yaolin. Land Information System [M]. Beijing: China Agriculture Press, 2003.M. Young, The Technical Writer's Handbook. Mill Valley, CA: University Science, 1989.

[7] Eisenhardt K M. Building theory from case study research [J]. Academy of management Review, 1989, 14: 532-550.

[8] Yin R K. Case Study Research: Design and Methods [M].3rd ed. Thousand Oaks: Sage, 2003.

[9] Li Xinxin, Lu Qiang. Chinese Management Research: Review and Evaluation [J]. Research Management, 2010, 31(5): 35-44+101.

[10] Song Yuwen, Yu Wei. Analysis of the Organizational Mode of Virtual Industry Clusters and Policy Suggestions [J]. China Science and Technology Forum, 2008, (11): 52-56.

[11] Gao Changyuan, Cheng Wei. Research on the Generation and Organization Mode of High-tech Virtual Industrial Clusters[J]. Science and Technology Progress and Policy, 2008, 28(1): 52-55. 